\title{
LINEAR DIFFERENTIAL EQUATIONS WITH EXCEPTIONAL FUNDAMENTAL SETS. II
}

\author{
NORBERT STEINMETZ
}

(Communicated by Clifford J. Earle, Jr.)

\begin{abstract}
We prove a sharp order estimate for entire functions of completely regular growth, whose zeros are distributed near finitely many rays $\arg z=\omega_{j}$ in terms of the angles $\omega_{j}$. This result then leads immediately to a proof of a conjecture of Hellerstein and Rossi concerning the distribution of zeros of the solutions of linear differential equations with polynomials coefficients.
\end{abstract}

\section{INTRODUCTION}

Let $w_{1}, w_{2}$ be linearly independent solutions of

$$
w^{\prime \prime}+p(z) w=0,
$$

where $p$ is a nonconstant polynomial. From the well-known results of Hille [6, Chapter 5] on the asymptotic distribution of zeros, Gundersen [4] deduced that the exponent of convergence of the nonreal zeros of $E=w_{1} w_{2}$ equals $1+\frac{1}{2} \operatorname{deg} p$. In an earlier paper, Hellerstein, Shen, and Williamson [5] showed that $E$ has infinitely many nonreal zeros.

The methods in $[4,5]$ do not apply to the $n$th order case. In this paper we prove an analogon of Gundersen's result for the solutions of $n$th order linear differential equations

$$
w^{(n)}+p_{n-2}(z) w^{(n-2)}+\cdots+p_{0}(z) w=0
$$

with polynomial coefficients, which is even slightly sharper. In particular, we solve Problem 2.72 in [1], posed by Hellerstein and Rossi.

This is done by proving a sharp order estimate (Theorem 1) for entire functions of completely regular growth, which seems to be of independent interest.

\section{Notation}

Let $E$ be an entire function of finite positive order $\lambda$ and denote the counting function of zeros of $E$ lying in the planar set $S$ by $N(r, 1 / E, S)$. We say that the zeros of $E$ are distributed near the rays

$$
\arg z=\omega_{j}, \quad 0 \leq \omega_{1}<\omega_{2}<\cdots<\omega_{k}<\omega_{k+1}=\omega_{1}+2 \pi,
$$

Received by the editors June 9, 1990 and, in revised form, December 12, 1990.

1980 Mathematics Subject Classification (1985 Revision). Primary 30D35, 34A20. 
if for every sufficiently small $\delta>0$,

$$
N\left(r, 1 / E, \omega_{j}+\delta<\arg z<\omega_{j+1}-\delta\right)=o\left(r^{\lambda}\right) \quad \text { as } r \rightarrow \infty
$$

$(j=1,2, \ldots, k)$.

The basic results in [8] for solutions of equation (2) can be summarized as follows:

Let $w$ be a transcendental solution of $(2)$, where $p_{0}, \ldots, p_{n-2}$ are polynomials. Then $w$ is a completely regular growing entire function of order $\lambda$ (in the sense of Lewin [7]), $0<\lambda<\infty$, whose zeros are distributed near finitely many rays (3).

\section{Results}

We first prove a result on completely regular growing entire functions with radially distributed zeros, from which the main result concerning the distribution of zeros of a fundamental set of (3) follows.

Theorem 1. Let $E$ be a completely regular growing entire function of order $\lambda$, whose zeros are distributed near the rays (3). Then either

$$
\lambda \leq \frac{\pi}{\min \left(\omega_{j+1}-\omega_{j}\right)}
$$

or else

$$
\delta(0, E)>0 .
$$

Remark. There is a similar result [3, Corollary 1.2]: If the zeros of the entire function $E$ are lying on the rays $\arg z=\omega_{j}$ and if the order of $E$ is finite, but sufficiently large (depending on the geometry of the rays), then $\delta(0, E)>0$. For us it is important to have an explicit (and sharp) bound.

Theorem 2. Let $\left\{w_{1}, w_{2}, \ldots, w_{n}\right\}$ be a fundamental set of $(2)$, where $p_{0}, \ldots$, $p_{n-2}$ are polynomials. Suppose that the zeros of $\left\{w_{1}, \ldots, w_{n}\right\}$ are distributed near the rays (3). Then either

$$
\lambda\left(w_{\nu}\right) \leq \frac{\pi}{\min \left(\omega_{j+1}-\omega_{j}\right)}, \quad \nu=1, \ldots, n,
$$

or else the coefficients in (2) are constants.

Remark. The product $E=w_{1} w_{2} \cdots w_{n}$ is a completely regular growing entire function of order $\lambda, 0<\lambda<\infty$, or a polynomial, and the radial distribution of the zeros of $\left\{w_{1}, \ldots, w_{n}\right\}$ corresponds to the function $E$ (and its order).

For the sake of completeness we reformulate Theorem 2 in two special cases.

Corollary 1. Let $\left\{w_{1}, \ldots, w_{n}\right\}$ be a fundamental set of (2), whose zeros are distributed near the rays $\arg z=2 j \pi / k \quad(1 \leq j \leq k, k \geq 2)$. Then

$$
\lambda\left(w_{\nu}\right) \leq k / 2, \quad \nu=1, \ldots, n .
$$

Corollary 2. Let $\left\{w_{1}, \ldots, w_{n}\right\}$ be a fundamental set of (2), whose zeros are distributed near the real axis. Then the coefficients in (2) are constants. 
Remark. This result has also been proven by Brüggemann [2] in a slightly weaker form.

\section{Proof of TheOREM 1}

Since the function $E$ has completely regular growth and since its zeros are distributed near the rays $\arg z=\omega_{j}$, there are complex numbers $c_{j}$ such that

$$
\log |E(z)|=\operatorname{Re}\left(c_{j} z^{\lambda}\right)+o\left(|z|^{\lambda}\right) .
$$

This is true as $|z| \rightarrow \infty$ outside a set $\mathscr{E} \subseteq(0, \infty)$ of linear density zero:

$$
\lim _{r \rightarrow \infty} \operatorname{mes}(\mathscr{E} \cap[0, r]) / r=0
$$

and uniformly in $\omega_{j}+\eta \leq \arg z \leq \omega_{j+1}-\eta, \eta>0$ arbitrary.

Thus the contribution of the sector $\omega_{j}<\arg z<\omega_{j+1}$ to $m(r, E)$ and $m(r, 1 / E)$ is asymptotically $M_{j} r^{\lambda}$ and $m_{j} r^{\lambda}$, respectively, where

$$
M_{j}=\frac{1}{2 \pi} \int_{\omega_{j}}^{\omega_{j+1}}\left[\operatorname{Re}\left(c_{j} e^{i \lambda \theta}\right)\right]^{+} d \theta
$$

and

$$
m_{j}=\frac{1}{2 \pi} \int_{\omega_{j}}^{\omega_{j+1}}\left[\operatorname{Re}\left(c_{j} e^{i \lambda \theta}\right)\right]^{-} d \theta
$$

(as usual $x^{+}=\max (0, x)$ and $x^{-}=\max (0,-x)$ ).

If we assume $\delta(0, E)=0$, then we must have $m_{j}=0 \quad(1 \leq j \leq k)$, but $M_{j} \neq 0$ for at least one $j$ since $E$ has order $\lambda$.

Thus $c_{j} \neq 0$ and $\left|c_{j}\right| \cos \left(\arg c_{j}+\lambda \theta\right) \geq 0$ in $\omega_{j}<\theta<\omega_{j+1}$ for at least one $j$, which gives

$$
\lambda\left(\omega_{j+1}-\omega_{j}\right) \leq \pi
$$

and so (7).

\section{Proof of Theorem 2}

Set $E=w_{1} w_{2} \cdots w_{n}$ and assume first that $E$ is transcendental of order $\lambda$. The Wronskian

$$
W=W\left(w_{1}, w_{2}, \ldots, w_{n}\right)
$$

is a constant $c$, say. Thus

$$
m\left(r, \frac{1}{E}\right)=m\left(r, \frac{W}{E}\right)+O(1)=O(\log r)
$$

by the lemma on the logarithmic derivative and so

$$
\lambda \leq \pi / \min \left(\omega_{j+1}-\omega_{j}\right)
$$

holds by Theorem 1 .

However, if $\lambda<\max \left(\lambda\left(w_{1}\right), \ldots, \lambda\left(w_{n}\right)\right)$, then by [9, Theorem 2] the coefficients of (2) are constants, and this is of course also true if $E$ is a polynomial. Thus, either $\lambda=\max \left(\lambda\left(w_{1}\right), \ldots, \lambda\left(w_{n}\right)\right)$ and (16) implies (7) or the coefficients of (2) are constants. 


\section{REFERENCES}

1. D. A. Brannan and W. K. Hayman, Research problems in complex analysis, Bull. London Math. Soc. 21 (1989), 1-35.

2. F. Brüggemann, On solutions of linear differential equations with real zeros; proof of a conjecture of Hellerstein and Rossi, Proc. Amer. Math. Soc. 113 (1991), 371-379.

3. A. Edrei, W. H. J. Fuchs, and S. Hellerstein, Radial distribution of the values of a meromorphic function, Pacific J. Math. 11 (1961), 135-151.

4. G. Gundersen, On the real zeros of solutions of $f^{\prime \prime}+A(z) f=0$ where $A(z)$ is entire, Ann. Acad. Sci. Fenn. Ser. A. I Math. 11 (1986), 275-294.

5. S. Hellerstein, L. C. Shen, and J. Williamson, Real zeros of derivatives of meromorphic functions and solutions of second-order differential equations, Trans. Amer. Math. Soc. 285 (1984), 759-776.

6. E. Hille, Lectures on ordinary differential equation, Wiley, New York, 1976.

7. B. J. Lewin, Nullstellenverteilung ganzer Funktionen, Akademie-Verlag, Berlin, 1962.

8. N. Steinmetz, Exceptional values of solutions of linear differential equations, Math. Z. 201 (1989), 317-326.

9. __ Linear differential equations with exceptional fundamental sets, submitted.

Institut für Mathematik, Universität Dortmúnd, Postfach 500 500, D-4600 Dortmúnd, GERMANY 